\title{
Luxación pura de facetas articulares T11-T12 sin secuelas neurológicas. Caso clínico
}

\author{
F. Ugarriza; L.M. Bernal-García; L.M. Lorenzana; M. Ortega-Martínez; L.F. Porras y J.M. Cabezudo
}

Servicio de Neurocirugía. Complejo Hospitalario Infanta Cristina. Badajoz.

Resumen

La luxación bilateral pura de facetas articulares (LFA) a nivel de la columna torácica, es una entidad rara pero que da lugar a graves lesiones medulares en un alto porcentaje de casos. En la gran mayoría de los casos se produce por un mecanismo de flexióndistraccion.

Presentamos el caso de un varón que sufrió un politraumatismo con importantes lesiones toraco-abdominales y esqueléticas, por lo que estuvo ingresado en un hospital comarcal, y al que, un mes después, se le diagnosticó una luxación bilateral de facetas articulares a nivel T11-T12, que en un principio pasó desapercibida. El diagnóstico se obtuvo mediante las radiografías simples y la TC con reconstrucción sagital. Fue intervenido quirúrgicamente para realizar la reducción de la luxación y fijación instrumentada a nivel T11-T12. La recuperación fue completa en la primera semana postoperatoria.

La LFA pura a nivel torácico, en un elevado porcentaje, se asocia a lesiones toracoabdominales que agravan el proceso y pueden contribuir a que las LFA pasen inadvertidas inicialmente, sobre todo en pacientes sin afectación neurológica, con riesgo de provocar un daño neurológico secundario. La TC con reconstrucción sagital nos permite realizar un diagnostico adecuado y precoz. El tratamiento es quirúrgico, tanto para la reducción como para la fijación posterior, ya que se trata de una lesión altamente inestable. El pronóstico es malo en pacientes con lesiones medulares completas, mientras que la mejoría es frecuente en los casos de lesión incompleta.

PALABRAS CLAVE: Luxación bilateral de facetas articulares. Traumatismo raquídeo. Columna torácica. TC con reconstrucción sagital.

Recibido: 09-05-05. Aceptado: 06-06-05
Bilateral pure T11-T12 facet dislocation without neurologic residual deficit

\section{Summary}

Bilateral pure facets dislocation in the thoracic spine is rare, but when it does occur in almost every istance produces a severe spinal cord lesion. It is thought to be caused by a flexion distraction mechanisms in most of the cases.

A case is presented of a male suffered a polytrauma with important thoracic, abdominal, and esqueletic injuries in a car accident. The patient was in a hospital during two weeks, and one month after the trauma a bilateral pure dislocation of T11-T12 facets was diagnosed. Diagnosis was made with radiographs and CT with sagittal reconstruction. After an open reduction and fixation, recovery was complete within the first postoperative week.

Facet dislocation in the thoracic spine is associated in many cases with thoracic and abdominal injuries that worsen the clinical picture, and thus it may contribute to misdiagnosis of the facet dislocation, specially in the cases with no neurological symptoms, adding a risk of secondary spinal cord damage. High-resolution CT with sagittal reconstruction provides an adequate and rapid demonstration of the luxation and associated thoraco-abdominal damages. Facet dislocation are unstable injuries that require open reduction and fixation. Recovery of patients with incomplete lesions is frequent, however, recovery from complete lesions did not occur.

Abreviaturas. CPL: complejo ligamentario posterior. FA: facetas articulares; LFA: luxación de facetas articulares. Proyección AP: proyección antero-posterior en la radiografia simple RM: resonancia magnética. Rx: radiografía simple. TC: tomografia computarizada. TCE: traumatismo craneoencefálico. UCI: unidad de cuidados intensivos. 


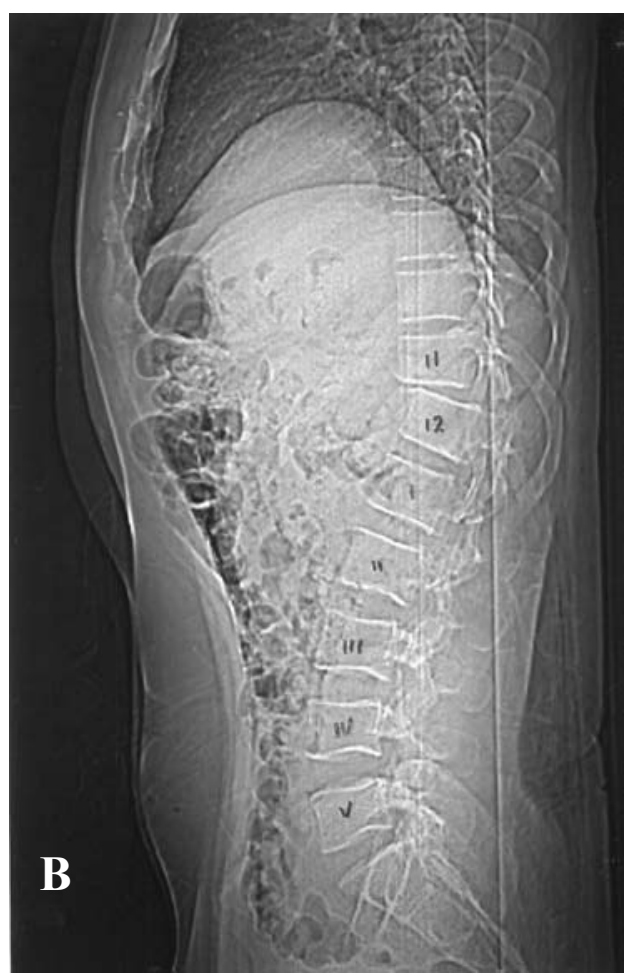

KEY WORDS: Bilateral facets dislocation. Spinal injury. Thoracic spine. CT sagittal reconstruction.

\section{Introducción}

La luxación traumática bilateral de facetas articulares (LFA) a nivel de la columna torácica es una patología infrecuente que debe ser diferenciada de la fractura-luxación. La principal característica en la LFA es que se trata de una lesión de tejidos blandos ${ }^{7}$, permaneciendo íntegros las facetas articulares (FA), muro posterior, pedículos, y láminas, aunque en ocasiones puede asociarse una pequeña fractura por compresión de la porción anterior del cuerpo vertebral inferior ${ }^{7}$, y suele provocar lesiones medulares graves en un elevado porcentaje de los $\operatorname{casos}^{2,4,5,6,7}$.

Presentamos un caso de LFA a nivel T11-T12, que pasó desapercibida en principio, con mínima afectación neurológica inicial, y una recuperación prácticamente inmediata tras la reducción abierta y fijación instrumentada.

\section{Caso Clínico}

Varón de 28 años de edad que ingresa en el servicio de urgencias de un hospital comarcal presentando, a consecuencia de un accidente de automóvil, un politraumatismo con fractura de $6^{\circ}$ arco costal y contusión pulmonar derecha, laceración renal con hematoma subcapsular y suprarrenal derechos, hematoma de pared abdominal, luxación de codo derecho y lesión ligamentaria en rodilla del mismo lado, heridas con pérdida de sustancia en ambas extremidades inferiores, y sin signos de TCE.

El paciente precisó intubación orotraqueal y ventilación asistida por insuficiencia respiratoria, y transfusiones sanguíneas por anemia aguda, ingresando en UCI. Extubado a las 48 horas sin incidencias, pasa a planta de hospitalización 24 horas después, donde permanece diez días, procediéndose a la colocación de un yeso en la extremidad inferior derecha.

Desde el alta hospitalaria, 13 días después del traumatismo, el paciente presenta dolor a nivel dorso-lumbar, achacado en principio al traumatismo renal, que en los últimos 7 días se asocia a sensación subjetiva de pérdida de fuerza con parestesias-disestesias en ambas extremidades inferiores, mas acusadas al permanecer acostado, $\mathrm{y}$ en las ultimas 72 horas dificultad para iniciar la micción y estreñimiento. El paciente acude, un mes después del traumatismo, al hospital comarcal donde se practica Rx de columna vertebral torácica y lumbar en la que se aprecia un aumento de la distancia interespinosa en la proyección anteroposterior, y en la lateral un desplazamiento anterior del cuerpo vertebral de T11 sobre T12, con una posible luxación de facetas articulares a este nivel, y una mínima fractura de la porción mas anterior del cuerpo vertebral de T12 (Fig. 1). Se practica RM de la zona en la que se descarta la existencia de hematomas en el canal, confirmándose los hallazgos de la $\mathrm{Rx}$, y con una dudosa imagen de 


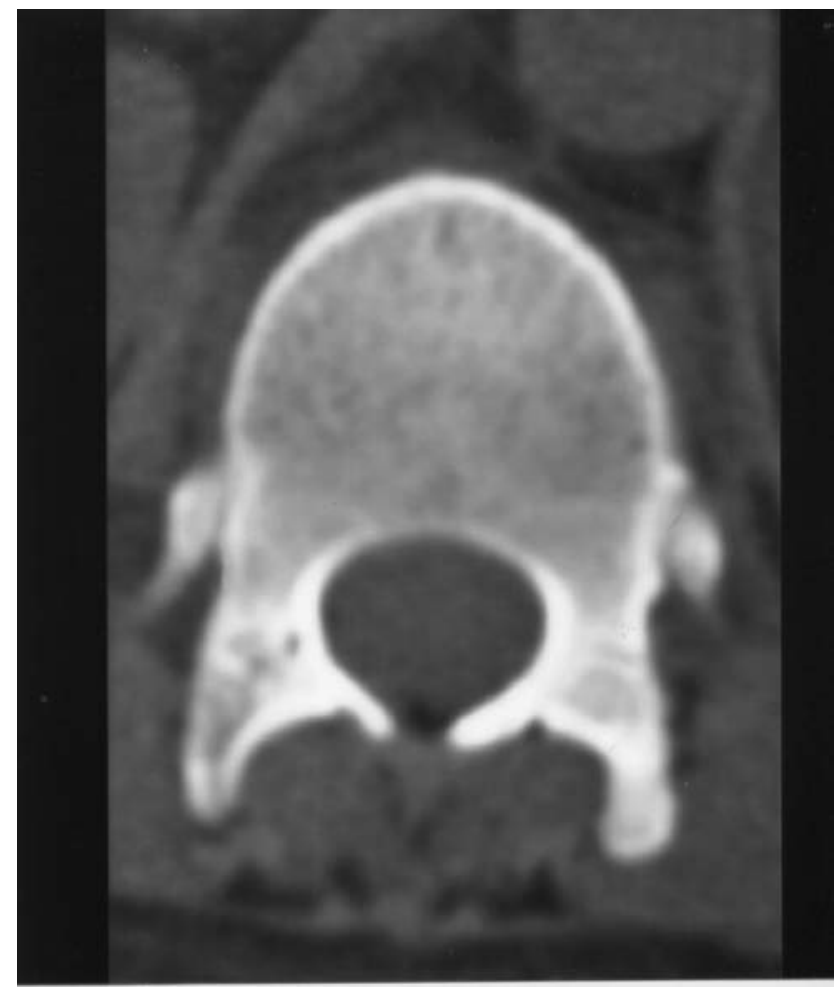

Figura 2. TC axial que muestra el signo de la faceta desnuda, viéndose la faceta superior de T12, y la falta de relación con la faceta inferior de T11. contusión medular. Así mismo se observa la presencia de sangre a nivel de ligamento interespinoso. El paciente es remitido a nuestro servicio.

En el momento de su ingreso el paciente presentaba una leve paraparesia $(4+/ 5)$ de predominio proximal, sin déficits sensitivos, leve hiperreflexia rotuliana bilateral simétrica, sin signos de liberación piramidal. Se practica $\mathrm{TC}$, que en los cortes axiales pone de manifiesto la existencia de una anormal disposición de las facetas T11-T12, con una estenosis del canal a ese nivel, la existencia de un signo de la faceta desnuda (Fig. 2), y en la reconstrucción sagital y tridimensional se confirma la luxación y bloqueo de las facetas articulares a nivel T11-T12 de forma bilateral (Fig. 3).

El paciente fue intervenido quirúrgicamente mediante un abordaje posterior, procediéndose a la extirpación de un magma cicatricial a nivel de ambas articulares, curetaje de las carillas de las FA superiores de T12, y reducción abierta de la luxación mediante maniobras de distracción-extensión, consiguiéndose una reducción casi completa tras practicar una laminectomía parcial bilateral, que facilitó la reducción, seguida de fijación T11-T12 con tornillos pediculares, barras, e injerto autólogo (Fig 4). El paciente evoluciona favorablemente desapareciendo su sintomatología neurológica en la primera semana, siendo dado de alta sin dolor y deambulando normalmente.
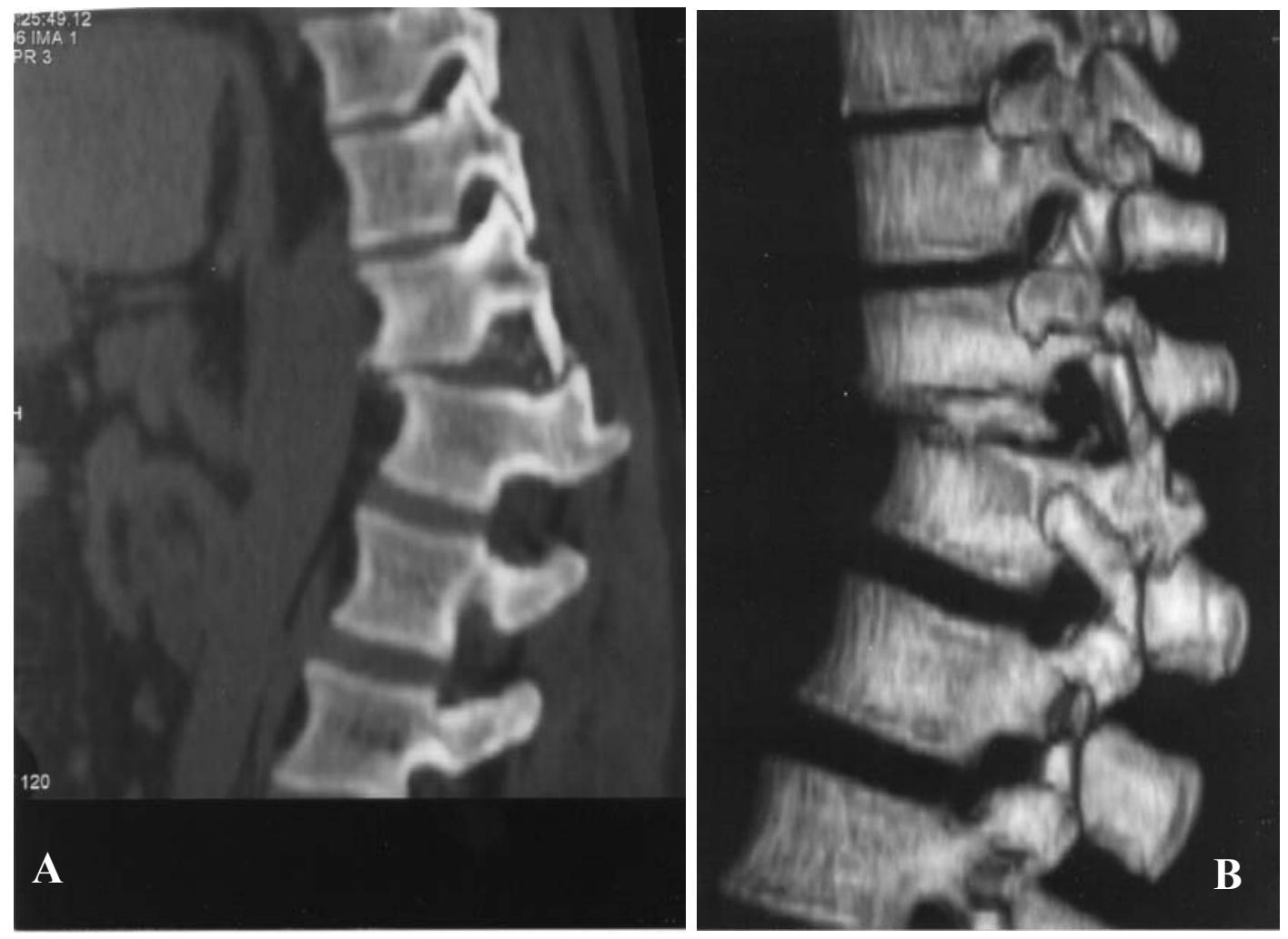

Figura 3. Reconstrucción sagital (A) $y$ tridimensional $(B)$ de la TC, mostrando la luxación y bloqueo de las facetas articulares. 


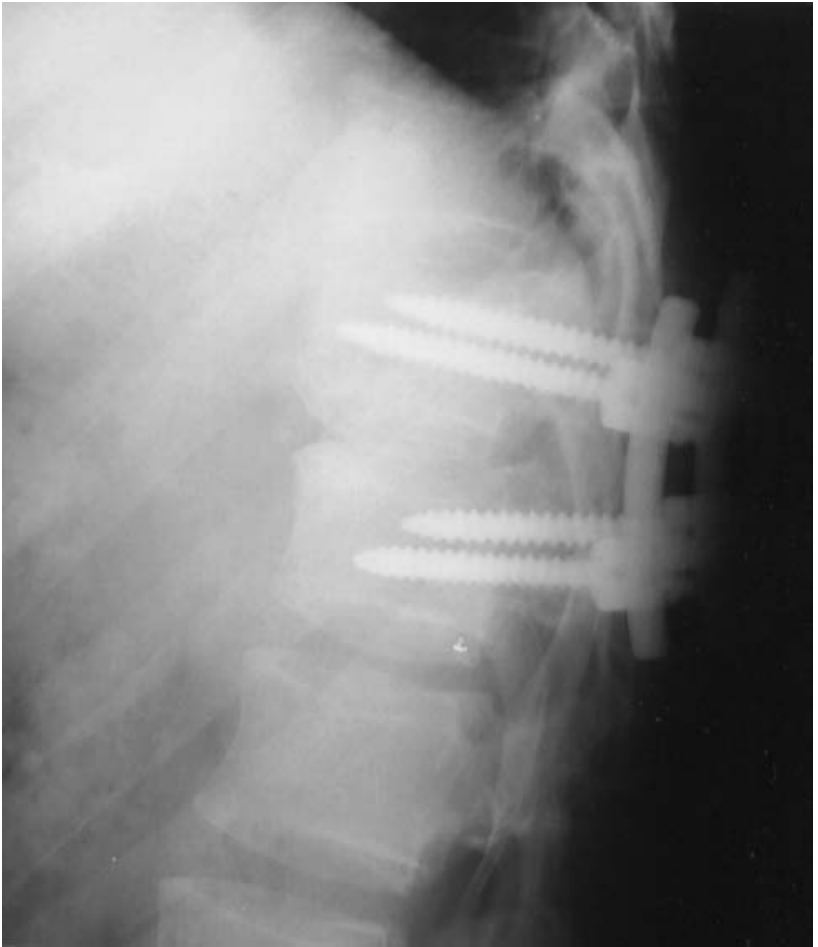

Figura 4. Rx postoperatoria inmediata en proyección lateral mostrando la reducción y fijación instrumentada del segmento T11-T12.

\section{Discusión}

Las LFA a nivel de la columna torácica, aunque raras, ocasionan una importante morbilidad, ya que dan lugar a paraplejías en el $62-89 \%$ de los casos, siendo el porcentaje de pacientes sin afectación neurológica sólo del $3-7 \%^{2,7}$. Su rareza es debida a la gran estabilidad de la columna torácica, la cual viene determinada por la disposición casi coronal de las FA, la limitación de la movilidad por la articulación de los arcos costales con el esternón y la columna vertebral, y la fortaleza del complejo ligamentario posterior (CLP) $)^{7,9,12}$.

Manaster ${ }^{9}$ diferenció tres tipos de LFA: la luxación lateral del cuerpo vertebral con bloqueo lateral de FA, la cifosis aguda con las FA luxadas superiormente, y la luxación anterior del cuerpo vertebral con bloqueo anterior de FA, grupo éste último en el que se incluiría el presente caso

\section{Mecanismos de producción}

La mayoría de los autores consideran que la LFA se produce a través de un mecanismo de flexión-distracción ${ }^{1,4,8,10,11,12,14,15}$. Asimismo, Denis ${ }^{1}$ remarcó que la ruptura del CLP por sí sola es insuficiente para que se produzca la LFA. Sólo cuando esta incompetencia se asocia a la rotura del anulus discal y del ligamento longitudinal posterior se podría producir la luxación.

Para que la LFA se produzca es necesario un traumatismo de gran energía, siendo la causa más frecuente los accidentes con vehículo de motor y las precipitaciones ${ }^{2,6,12}$. Es por ello que el 20-50\% de los casos se asocian a lesiones viscerales y/o vasculares a nivel toraco-abdominal ${ }^{7,13}$. Este hecho, además de agravar el proceso, hace que en ocasiones la LFA pase inadvertida, sobre todo en pacientes sin afectación neurológica inicial, como sucedió en nuestro caso, con el consiguiente riesgo de daño neurológico secundario. Por lo que, en este tipo de pacientes en los que hay patología toracoabdominal, sería recomendable la realización de un TC que permita diagnosticar las lesiones pulmonares o abdominales, así como las raquídeas.

Exceptuando la columna cervical, el 51-60\% de las LFA se producen a nivel de la charnela toraco-lumbar ${ }^{7,8}$. Esto se debe a la existencia de las costillas flotantes a este nivel, con la consiguiente falta de sujeción de la caja costal, la transición cifosis-lordosis con la diferente disposición de las FA, y la mayor movilidad del segmento lumbar?

\section{Diagnóstico}

El estudio radiológico simple nos permite visualizar en la proyección lateral el desplazamiento del cuerpo superior sobre el inferior, manteniendo integro el muro posterior, la luxación de las FA, y en ocasiones pequeñas fracturas de la porción más anterior del cuerpo anterior. En la proyección AP es característica la existencia de un aumento de la distancia interespinosa al nivel afecto $^{2}$, mientras que, a diferencia de las fracturas-aplastamiento del cuerpo vertebral, la distancia entre pedículos se mantiene ${ }^{7}$. Sin embargo, un alto número de ellas pueden pasar desapercibidas en el estudio simple, sobre todo a nivel torácico medio y alto alt, $^{2,12}$, ya que las costillas pueden dificultar la visualización de la luxación.

La RM, aunque aporta poco al diagnóstico de la LFA, nos permite la visualización del canal, y el diagnóstico de posibles lesiones medulares traumáticas, hematomas dentro del canal, lesiones discales, lesiones de los ligamentos inter y supraespinosos y la musculatura paravertebral, que pueden asociarse ${ }^{3}$.

La TC constituye la mejor arma diagnóstica en la LFA $^{7,9,12}$. En los cortes axiales podemos ver la anormal disposición de las facetas articulares, siendo característico el denominado signo de la faceta desnuda ${ }^{1,2,3}$, descrito por O'Callaghan $^{11}$, así como en ocasiones la imagen de doble vértebra. No obstante, la reconstrucción sagital es de suma importancia ya que es la que permite confirmar la existencia de la luxación y posible bloqueo de las FA, y nos permite medir el diámetro del canal medular, que a diferencia de otro tipo de traumas, parece tener importancia en estas lesiones, con muy mal pronóstico en los casos en los que el 
diámetro del canal sea menor de $10 \mathrm{~mm}^{2,3,7}$.

\section{Tratamiento}

El tratamiento es quirúrgico ya que, además de que la reducción cerrada es altamente improbable, la LFA provoca una gran inestabilidad por lo que es mandatario la fijación instrumentada ${ }^{3,7,9,12}$. Con la intervención quirúrgica se persiguen tres objetivos: la reducción de la luxación, la adecuada descompresión medular, y la estabilización y fusión del segmento inestable.

El abordaje debe de ser posterior, estando contraindicado el abordaje anterior ya que además de la gran dificultad que entraña realizar la reducción por está vía, aumentamos la inestabilidad ${ }^{7,10}$. Parece lógico pensar que dado que la LFA se ha producido por un mecanismo de flexión-distracción, la fijación deba realizarse en compresión con la columna en extensión ${ }^{2,7,10}$. Sin embargo, algunos autores consideran que la compresión puede ocasionar una extrusión del disco intervertebral, provocando nuevas compresiones medulares, por lo que creen que sólo debería realizarse en pacientes con lesiones neurológicas completas, estando indicada la fijación en distracción para los casos sin afectación neurológica o cuando esta sea parcial ${ }^{2,7}$. Sin embargo, la utilización de registro neurofisiológico intraoperatorio, nos da un amplio margen de seguridad para realizar la compresión.

\section{Pronóstico}

El pronóstico en cuanto a la recuperación neurológica es razonablemente predecible. Aquellos pacientes con lesión neurológica completa permanecen en este estado tras la descompresión, mientras que la mayoría de los que presentan una lesión incompleta mejoran de forma manifiesta tras la cirugía 7 . Por ello, estos pacientes deben ser intervenidos de forma urgente para evitar el alto riesgo de deterioro neurológico.

\section{Conclusiones}

La LFA pura a nivel torácico constituye una entidad rara pero con un alto índice de lesiones medulares. En un elevado porcentaje se asocian lesiones toraco-abdominales que agravan el proceso y pueden contribuir a que las LFA pasen inadvertidas inicialmente, y ocasionar un daño neurológico secundario. La TC con reconstrucción sagital nos permite realizar un diagnostico adecuado y precoz. El tratamiento es quirúrgico, estando indicada la vía posterior tanto para la reducción como para la descompresión y fijación instrumentada de una lesión raquídea altamente inestable.

\section{Bibliografía}

1. Denis, F.: The three columns spine and its significance in the classification of acute thoracolumbar spinal injuries. Spine 1983; 8: 817-832.

2. Gellad, F.G., Levine, A.M., Joslyn, J.N., Edwards, C.C., Bosse, M.: Pure thoracolumbar facet dislocation: Clinical features and CT appearance. Radiology 1986; 161: 505-508.

3. Gharib, A., Postel, G., Mirza, S., Mann, F.A.: A thoracic spine translation injury with lateral facet dislocation. A J R 2002; 178: 1450 .

4. Holdsworth, F.: Fractures, dislocations and fracturedislocations of the spine. J Bone Joint Surg (Am) 1970; 60: 1535-1551.

5. Jackson, R.H., Quisling, R.G., Day, A.L.: Fracture and complete dislocation of the thoracic or lumbosacral spine: Report of three cases. Neurosurgery 1979; 5: 250-253.

6. Korovessis, P., Sidiropoulos, P., Dimas, A.: Complete fracture-dislocation of the thoracic spine without neurologic deficit: Case report. J Trauma 1994; 36: 122-124.

7. Levine, A.M., Bosse, M., Edwards, C.C.: Bilateral facet dislocations in the thoracolumbar spine. Spine 1988; 13: 630-640.

8. Lewis, J., McKibbin, B.: The treatment of unstable fracture dislocation of the thoracolumbar spine accompanied by paraplegia. J Bone Joint Surg 1974; 56B: 603-612.

9. Manaster, B.J., Osborn, A.G.: CT patterns of facet fracture dislocations in the thoracolumbar region. A J R 1987; 148: 335-340.

10. McAfee, P.D., Hansen, A.V., Fredrickson, B.E., Lubicky, J.P.: The value of computed tomography in thoracolumbar fractures. J Bone Joint Surg 1983; 65A: 456-473.

11. O'Callaghan, J.P., Ullrich, C.G., Yuan, H.A. Kieffer, S.A.: CT of facet distraction in flexion injuries of the thoracolumbar spine: the "nacked" facet. A J N R 1980; 1: 97-102.

12. Sharafuddin, M.J.A., Hitchon, P.W., El-Khoury, G.Y., Dyste, G.N.: Locked facets in the thoracic spine: report of three cases and review. J Spine Disord 1990; 3: 255- 258.

13. Singh, K., Vaccaro, A.R., Eichenbaum, M.D., Fitzhenry, L.N.: The surgical management of thoracolumbar injuries. J Spine Cord Med 2004; 27: 95-101.

14. Whitesides, T.E.: Traumatic kyphosis of the thoracolumbar spine. Clin Orthop 1979; 128: 78- 92.

15. Willems, M.H.A., Braakman, R., Van Linge, B.: Bilateral locked facets in the thoracic spine. Acta Orthop Scand 1984; 55: 300-303.

Ugarriza, F.; Bernal-García, L.M.; Lorenzana, L.M.; OrtegaMartínez, M.; Porras, L.F.; Cabezudo, J.M.: Luxación pura de facetas articulares T11-T12 sin secuelas neurológicas. Cso clínico. Neurocirugía 2006; 17: 261-266.

Correspondencia postal: Fernando Ugarriza. c/ Castillo de Rena 43. Puerta 2. 6006 Badajoz. 
Comentario del trabajo Luxación pura de facetas articulares T11-T12 sin secuelas neurológicas de Ugarriza y cols

Ugarriza y cols presentan un caso de luxación pura de facetas articulares T11-T12 cuya principal peculiaridad radica en la ausencia de secuelas neurológicas. Este hecho, aunque poco frecuente, ha sido ya descrito en la literatura. Gellad y cols. ${ }^{1}$, en una serie de 29 luxaciones facetarias puras toracolumbares, reportan un $17 \%$ de lesiones incompletas y un $7 \%$ de pacientes neurológicamente intactos.

Los autores tratan la luxación mediante fijación posterior transpedicular, realizando maniobra de distracción. Esta maniobra presenta algunas ventajas, pero también inconvenientes, respecto de la maniobra de compresión. Por una parte, la compresión presenta una mayor estabilidad aumentando las posibilidades de fusión. Pero por otra parte, la compresión crea una tensión sobre el disco que puede conducir a su extrusión posterior, con el consiguiente riesgo de afectación medular. Por ello, en lesiones neurológicas incompletas como la presentada en este caso, las cuales tienen una potencial capacidad de recuperación, parece prudente la distracción a fin de evitar posibles daños medulares secundarios ${ }^{2}$.

Los autores describen una interesante y poco frecuente lesión traumática espinal realizando una minuciosa revisión de la literatura. El manejo quirúrgico del paciente nos parece el adecuado dadas las características de la lesión.

\section{I.Arrese \\ J.F.Alén \\ Madrid}

\section{Bibliografía}

1. Gellad, F.E., Levine, A.M., Joslyn, J.N., Edwards, C.C., Bosse, M.: Pure thoracolumbarfacet dislocation: clinical features and CT appearance. Radiology 1986; 161: 505-508.

2. Levine, A.M., Bosse, M., Edwards, C.C.: Bilateral facets dislocations in the thoracolumbar spine Spine 1988; 13: 630-640. 